\title{
Loewner Approach Model Order Reduction in Hybrid BI-FEM Solution for the Design of Frequency Selective Surfaces
}

\author{
Valentín de la Rubia, Sofía Tinoco-Galafate and Zhen Peng
}

\begin{abstract}
A Loewner matrix data-driven model order reduction technique for fast frequency sweep in full-wave solution in large finite frequency selective surfaces is detailed. A hybrid Boundary Integral-Finite Element Domain Decomposition Method is addressed to accurately predict the electromagnetic behavior of these structures. A reduced-order model is built up by means of simulation scatering parameters. A Loewner matrix approach is taken into account to provide a tangential interpolation to output data. As a result, a dynamical system representation fitting the simulation data is obtained, and further electromagnetic insight can be obtained from this system representation.
\end{abstract}

Index Terms-Boundary element methods, discontinuous Galerkin methods, finite element methods, Loewner framework, reduced order systems.

\section{INTRODUCTION}

Frequency selective surfaces (FSS) [1] take part in many electromagnetic devices. Full-wave solution of electrically large finite Frequency Selective Surfaces (FSS) is a challenging numerical problem that can be addressed by a hybrid Boundary Integral-Finite Element Method (BI-FEM) Domain Decomposition approach [2], [3], [4]. It should be pointed out that an appropriate preconditioner must be applied for accurate solution of the large electromagnetic problem at its corresponding operating frequency.

The computational effort taken into account for a single frequency analysis is already large enough, however, a specific electrical behavior in a given frequency band is required for engineering purposes. In order to achieve this goal, multiple analyses have to be carried out until the final design is obtained. In order to compute the frequency behavior of these electrically large structures, a reduced-order model is desireable instead of computationally expensive frequency point analyses. Model order reduction stands upon replacing a rather complex mathematical model by a much simpler approximated one that still maintains certain aspects of the original model [5].

In this solution setting, the frequency dependency in the preconditioned numerical matrix operators to be solved is complex enough to apply standard model order reduction techniques, such as Asymptotic Waveform Expansion (AWE),
Padé via Lanczos (PvL), Proper Orthogonal Decomposition (POD) or Reduced Basis Method (RBM) [6], [7], [8], [9], where a reduced-order projection space is appropriately built up taking advantage of the specific parameter dependence. As a result, we propose to build up a reduced-order model by the only means of the output scattering data provided by the hybrid BI-FEM solver. This solver will be therefore used as a black box, unlike other model order reduction techniques, which gives rise to a data-driven model order reduction.

This data-driven reduced-order model is based on the Loewner matrix approach. This technique builds a linear dynamical system fitting the data and, not only the frequency band behavior of the FSS is determined, but also, we place special emphasis in getting a linear dynamical system description of the physics in the FSS such that we can use this information for design purposes.

\section{PRoblem Statement}

The time-harmonic Maxwell's equations can be written in variational form over an appropriate admissible discrete function space $\mathcal{X}_{h}$, viz.

$$
\begin{gathered}
\text { Find } E \in \mathcal{X}_{h} \text { such that } \\
a(E, v)=f(v) \quad \forall v \in \mathcal{X}_{h} .
\end{gathered}
$$

where $a(\cdot, \cdot)$ is an appropriate bilinear form taken the hybrid BI-FEM Domain Decomposition discretization of Maxwell's equations and $f(\cdot)$ is a linear functional. For a detailed definition of these forms, field variables and approximation space we refer to [2], [3], [4].

Taking frequency as a parameter, we are interested in solving the following discrete variational problem:

$$
\begin{gathered}
\text { Find } E(\omega) \in \mathcal{X}_{h} \text { such that } \\
a(E(\omega), v ; \omega)=f(v ; \omega) \quad \forall v \in \mathcal{X}_{h} .
\end{gathered}
$$

for every frequency $\omega$ in a given frequency band of interest $\left[\omega_{\min }, \omega_{\max }\right] \subset \mathbb{R}$. Nevertherless, we go a step further and we just need the input-output relation as a function of frequency, namely, the transfer function behavior of the electromagnetic structure. 


\section{A. Loewner Matrix Approach}

Let us denote the frequency domain $\mathrm{S}$ parameter data provided by BI-FEM Domain Decomposition solver as $S(s)$, where the complex frequency variable $s$ will be sampled in a specific frequency band. $S\left(s_{i}\right)$ stands for the matrix-valued transfer function, i.e., $S$ parameters, sampled at complex frequency $s_{i}$. The goal is to fit a descriptor system state space model of the form

$$
\Sigma:\left\{\begin{array}{rl}
E \frac{d}{d t} x(t) & =A x(t)+B u(t) \\
y(t) & =C x(t)
\end{array} .\right.
$$

$\Sigma$ is a linear dynamical system with $m$-inputs, $m$-outputs and $N$ dimensional internal variable, where $u(t)$ is the input, $y(t)$ is the corresponding output and $x(t)$ is the internal variable. $E, A \in \mathbb{C}^{N \times N}, B \in \mathbb{C}^{N \times m}$ and $C \in \mathbb{C}^{m \times N} . E$ matrix may be singular. The transfer function for the linear dynamical system (3) is

$$
\mathcal{S}(s)=C(s E-A)^{-1} B .
$$

The Loewner matrix approach builds a state space model $[E, A, B, C]$ up fitting the $\mathrm{S}$ parameter data $S\left(s_{i}\right)$ [10], [11].

\section{B. Tangential Interpolation}

Let us take into account the following frequency points in the complex plane $P=\left\{s_{1}, \ldots, s_{p}\right\}$, and its corresponding matrix-valued transfer function evaluations $\left\{H\left(s_{1}\right), \ldots, H\left(s_{p}\right)\right\} . P$ can be divided into two groups

$$
P=\left\{\lambda_{1}, \ldots, \lambda_{k}\right\} \cup\left\{\mu_{1}, \ldots, \mu_{h}\right\} .
$$

The rational interpolation method gives rise to a controllable and observable state space model from the sampled data $S\left(s_{i}\right)$ provided by the FEM analysis of microwave circuits. The tangential interpolation method uses the following right interpolation data

$$
\begin{gathered}
\Lambda=\operatorname{diag}\left\{\lambda_{1}, \ldots, \lambda_{k}\right\} \in \mathbb{C}^{k \times k} \\
R=\left[r_{1}, \ldots, r_{k}\right] \in \mathbb{C}^{m \times k} \\
W=\left[w_{1}, \ldots, w_{k}\right] \in \mathbb{C}^{m \times k}
\end{gathered}
$$

and the left interpolation data

$$
\begin{gathered}
M=\operatorname{diag}\left\{\mu_{1}, \ldots, \mu_{h}\right\} \in \mathbb{C}^{h \times h} \\
L=\left[\begin{array}{c}
l_{1} \\
\vdots \\
l_{h}
\end{array}\right] \in \mathbb{C}^{h \times m}, V=\left[\begin{array}{c}
v_{1} \\
\vdots \\
v_{h}
\end{array}\right] \in \mathbb{C}^{h \times m} .
\end{gathered}
$$

$r_{i}$ and $l_{j}$ stand for right and left tangential directions respectively. The tangential interpolation method sets a minimal dynamical system realization $[E, A, B, C]$ up such that the corresponding transfer function $\mathcal{S}(s)$ satisfies the following right and left constraints

$$
\begin{aligned}
& \mathcal{S}\left(\lambda_{i}\right) r_{i}=S\left(\lambda_{i}\right) r_{i}=w_{i} \\
& l_{j} \mathcal{S}\left(\mu_{j}\right)=l_{j} S\left(\mu_{j}\right)=v_{j}
\end{aligned}
$$

Loewner matrix and shifted Loewner matrix are specially well-suited to face this tangential interpolation problem since these matrices, by construction, give rise to a system realization fitting the simulation data [10]. The Loewner matrix of $S(s)$ can be built as

$$
\mathcal{L}=\left(\begin{array}{ccc}
\frac{v_{1} r_{1}-l_{1} w_{1}}{\mu_{1}-\lambda_{1}} & \ldots & \frac{v_{1} r_{k}-l_{1} w_{k}}{\mu_{1}-\lambda_{k}} \\
\vdots & \ddots & \vdots \\
\frac{v_{h} r_{1}-l_{h} w_{1}}{\mu_{h}-\lambda_{1}} & \ldots & \frac{v_{h} r_{k}-l_{h} w_{k}}{\mu_{h}-\lambda_{k}}
\end{array}\right)
$$

Similarly, the shifted Loewner matrix can be obtained by setting the Loewner matrix of $s S(s)$

$$
\sigma \mathcal{L}=\left(\begin{array}{ccc}
\frac{\mu_{1} v_{1} r_{1}-\lambda_{1} l_{1} w_{1}}{\mu_{1}-\lambda_{1}} & \ldots & \frac{\mu_{1} v_{1} r_{k}-\lambda_{k} l_{1} w_{k}}{\mu_{1}-\lambda_{k}} \\
\vdots & \ddots & \vdots \\
\frac{\mu_{h} v_{h} r_{1}-\lambda_{1} l_{h} w_{1}}{\mu_{h}-\lambda_{1}} & \ldots & \frac{\mu_{h} v_{h} r_{k}-\lambda_{k} l_{h} w_{k}}{\mu_{h}-\lambda_{k}}
\end{array}\right)
$$

Finally, recalling fundamental results from Loewner framework, cf. [11], the function

$$
S(s)=W(\sigma \mathcal{L}-s \mathcal{L})^{-1} V
$$

interpolates the $S$ paremeter simulation data.

\section{NUMERICAL RESULTS}

In this section, we apply the Loewner matrix approach described earlier to a Frequency Selective Surface made out of a finite size PEC where $8 \times 8$ circular rings are taking into account to build up a stop-band FFS. The dimension of the finite FSS is detailed in Fig. 1. The geometry details of the circular rings for each of the 64 unit cells are shown in Fig. 2. The hybrid BI-FEM Domain Decomposition solver is used to provide $S$ parameter data within the $5-20 \mathrm{GHz}$ band. Fig. 3 compares the full-wave response and the Loewner response. Good agreement in the $\mathrm{S}$ parameter behavior is found in the stop-band. Fig 4 detail another example unit cell, namely the unit cell in a $8 \times 8$ Swiss cross dipoles FSS where the cross is $10 \mathrm{~mm}$ long and $1 \mathrm{~mm}$ wide. Fig. 5 compares the results obtained by the full-wave solver and Loewner approach. Good agreement is once again found.

\section{CONCLUSION}

A Loewner matrix data-driven model order reduction technique for fast frequency sweep in full-wave solution in large finite frequency selective surfaces has been detailed. A hybrid Boundary Integral-Finite Element Domain Decomposition Method has been used as a black box to provide S parameter data. The Loewner matrix approach has been used for tangential interpolation of the simulation data. Finally, a minimal dynamical system realization has been shown to provide good accuracy results in the frequency band of interest.

\section{REFERENCES}

[1] B. A. Munk, Frequency selective surfaces. New York, NY: John Wiley \& Sons, 2000.

[2] Z. Peng, R. Hiptmair, Y. Shao, and B. Mackie-Mason, "Domain decomposition preconditioning for surface integral equations in solving challenging electromagnetic scattering problems," IEEE Trans. Antennas Propagat., vol. 64, no. 1, pp. 210-223, Jan. 2016. 


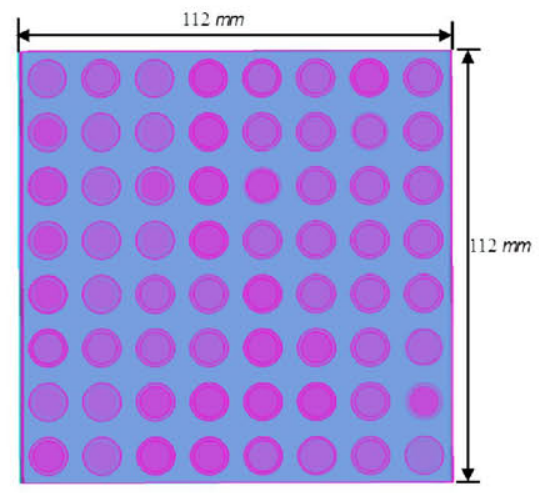

Fig. 1. $8 \times 8$ circular slot FSS.

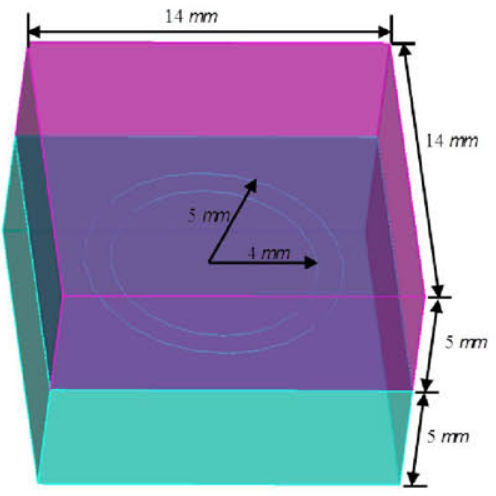

Fig. 2. Unit cell circular ring geometry in the FSS.

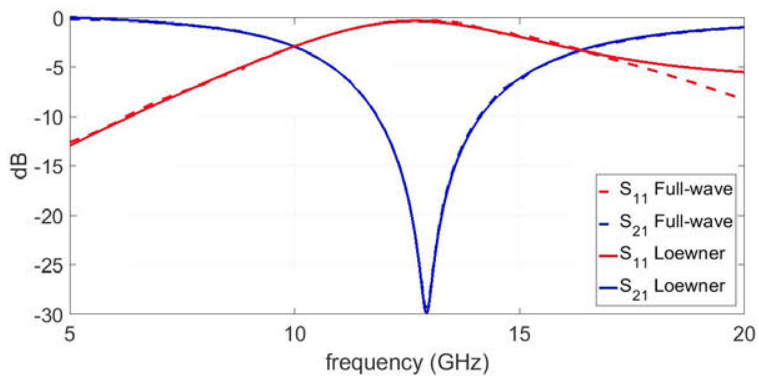

Fig. 3. Wide-band $\mathrm{S}$ parameter response comparison between data-driven reduced-order model and full-wave hybrid BI-FEM Domain Decomposition analysis in the circular ring FSS.

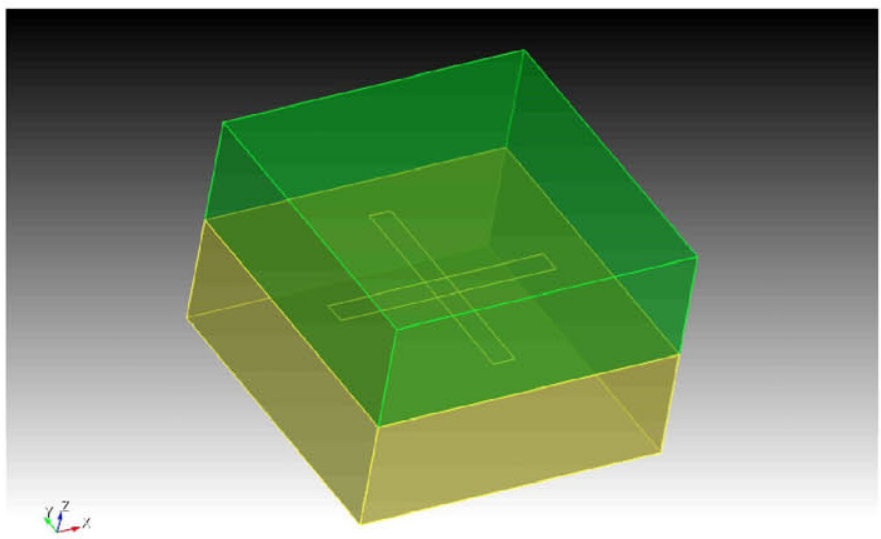

Fig. 4. $14 \times 14 \mathrm{~mm}$ unit cell Swiss cross dipole in the FSS.

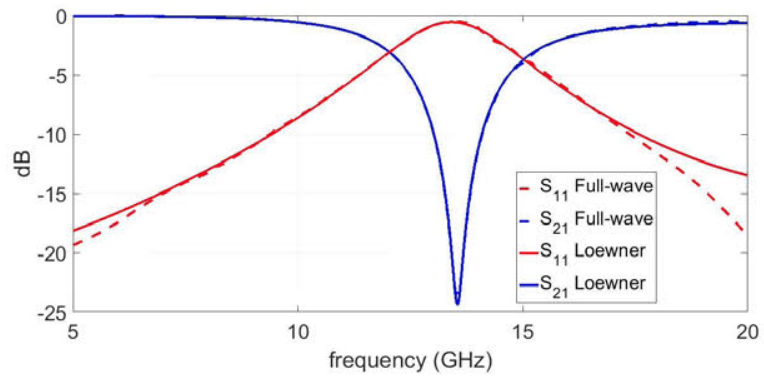

Fig. 5. Wide-band $\mathrm{S}$ parameter response comparison between data-driven reduced-order model and full-wave hybrid BI-FEM Domain Decomposition analysis in the Swiss cross dipole FSS.

[3] Z. Peng, K.-H. Lim, and J.-F. Lee, "Computations of electromagnetic wave scattering from penetrable composite targets using a surface integral equation method with multiple traces," IEEE Trans. Antennas Propagat., vol. 61, no. 1, pp. 256-270, Jan. 2013.

[4] Z. Peng and J.-F. Lee, "Non-conformal domain decomposition method with mixed true second order transmission condition for solving large finite antenna arrays," IEEE Trans. Antennas Propagat., vol. 59, no. 5, pp. 1638-1661, May 2011.

[5] A. C. Antoulas, Approximation of Large-Scale Dynamical Systems. Philadelphia, PA: SIAM, 2005.

[6] R. D. Slone, R. Lee, and J.-F. Lee, "Well-conditioned asymptotic waveform evaluation for finite elements," IEEE Trans. Antennas Propagat., vol. 51, no. 9, pp. 2442-2446, Sep. 2003.

[7] A. Sommer, O. Farle, and R. Dyczij-Edlinger, "Certified dual-corrected radiation patterns of phased antenna arrays by offlineonline order reduction of finite-element models," J. Comput. Phys., vol. 299, pp. 22-44, 2015.

[8] M. Rewienski, G. Fotyga, A. Lamecki, and M. Mrozowski, "Automated reduced model order selection," IEEE Antennas Wireless Propagat. Lett., vol. 14, pp. 382-385, 2015.

[9] V. de la Rubia, U. Razafison, and Y. Maday, "Reliable fast frequency sweep for microwave devices via the reduced-basis method," IEEE Trans. Microwave Theory Tech., vol. 57, no. 12, pp. 2923-2937, Dec. 2009.

[10] A. J. Mayo and A. C. Antoulas, "A framework for the solution of the generalized realization problem," Linear Algebra and its Applications, no. 425, pp. 634-662, Aug. 2007.

[11] S. Lefteriu and A. C. Antoulas, "A new approach to modeling multiport systems from frequency-domain data," IEEE Trans. Computer-Aided Design, vol. 29, no. 1, pp. 14-27, Jan. 2010. 